\title{
ESTADO NUTRICIONAL, ALIMENTACẼO E SAÚDE ORAL EM IDOSOS DE UM MUNICÍPIO DA SERRA GAÚCHA
}

\author{
Aline Piccoli Menin ${ }^{1}$ \\ Gabriela Néspolo² \\ Neide Maria Bruscato ${ }^{3}$ \\ Emílio Hideyuki Moriguchi4 \\ Juliana Rombaldi Bernardi5 \\ Josiane Siviero ${ }^{6}$
}

1 Acadêmica do curso de Nutrição da Faculdade Cenecista de Bento Gonçalves - FACEBG. E-mail: aline.menin@hotmail.com.

2 Acadêmica do curso de Nutrição da Faculdade Cenecista de Bento Gonçalves - FACEBG. E-mail: gabi.nespolo@outlook.com.

3 Graduada em Nutrição. Mestre em Medicina e Ciências da Saúde. Coordenadora Operacional do Projeto Veranópolis Estudos em Envelhecimento, Longevidade e Qualidade de Vida. E-mail: nmbruscato@yahoo.com.br.

4 Graduado em Medicina. Pós-doutorado em Metabolismo de Lipoproteínas e Doenças Ateroscleróticas. Professor do Departamento de Medicina Interna da Faculdade de Medicina da Universidade Federal do Rio Grande do Sul - UFRGS. Professor do Programa de Pós-Graduação em Saúde Coletiva da UNISINOS. Coordenador do Núcleo de Geriatria e Gerontologia do Hospital Moinhos de Vento. Sócio-Colaborador da Instituição Acadêmica Veranense de Educação em Saúde - AVAES. E-mail: emilio.moriguchi@gmail.com.

5 Graduada em Nutrição. Doutora em Saúde da Criança e do Adolescente. Professora Adjunta da Universidade Federal do Rio Grande do Sul - UFRGS, vinculada ao Departamento de Nutrição. E-mail: juliana.bernardi@yahoo.com.br.

6 Graduada em Nutrição. Doutora em Clínica Médica e Ciências da Saúde com ênfase em Geriatria. Professora da Universidade de Caxias do Sul - UCS e Faculdade Cenecista de Bento Gonçalves FACEBG. E-mail: josianesiviero@hotmail.com. 
resumo

O objetivo deste estudo foi avaliar o estado nutricional, alimentação e a saúde oral de idosos de um município da serra no Rio Grande do Sul, Brasil, por meio de um estudo transversal descritivo e analítico quantitativo, com dados primários. As variáveis analisadas foram: gênero; idade ( $\geq 60$ anos); peso (kg); altura (m); índice de massa corporal ( $\left(\mathrm{MC}=\mathrm{kg} / \mathrm{m}^{2}\right)$, circunferência da cintura $(\mathrm{CC}=\mathrm{cm})$, circunferência do braço $(\mathrm{CB}=\mathrm{cm})$ e circunferência da panturilha $(\mathrm{CP}=\mathrm{cm})$. Para avaliação do estado nutricional utilizou-se a Mini Avaliação Nutricional (MAN) e o consumo alimentar foi avaliado pelo Questionário de Frequência Alimentar (QFA). Em relação à saúde bucal, aplicou-se o GOHAl (Geriatric Oral Health Assessment Index) e o EAT-10 (Instrumento de Avaliação da Alimentação). Dos 135 idosos avaliados, 71,9\% eram mulheres. A obesidade abdominal foi prevalente nas mulheres $(p=0,037)$ e o risco de desnutrição pela MAN quanto à prevalência de baixo peso pelo IMC aumentaram com a idade ( $p=0,021$ e $p=0,039)$. Para os demais métodos de avaliação nutricional, as diferenças não foram estatisticamente significativas. Ainda pela MAN, houve diferença significativa, onde as mulheres se perceberam com melhor saúde ( $p=0,030)$. Ao investigar a saúde oral, não houve diferença significativa ( $p>0,05)$. 0 consumo de frutas frescas $(p=0,006)$ foi maior nas mulheres e batata/salgados fritos ( $p=0,001)$ foi mais consumido pelos homens. Não houve associação significativa entre os resultados da saúde oral com o consumo alimentar (p>0,05). Torna-se necessário reforçar orientações sobre alimentação saudável, para que os idosos possam adequar sua alimentação e prevenir problemas advindos de uma má nutrição, bem como, outros estudos devem ser realizados com estas variáveis.

palavras-chave

Estado nutricional. Alimentação. Saúde oral. Consumo alimentar.

O número de idosos vem apresentando rápido crescimento em relação à população mundial, principalmente nos países em desenvolvimento. Entre o ano de 2000 e 2050, a proporção de indivíduos com mais de 60 anos de idade entre a população mundial irá duplicar, passando de 11\% para 22\% (WHO, 2012). A população brasileira está envelhecendo de forma rápida; estima-se 
que existam atualmente no Brasil cerca de 23,5 milhões de pessoas com mais de 60 anos, representando 12,11\% da população brasileira. De acordo com a Organização Mundial de Saúde, o Brasil será o sexto país do mundo com o maior número de pessoas nesta faixa etária no ano de 2050, abrangendo 22,7\% dos habitantes brasileiros (IBGE, 2002).

No Rio Grande do Sul, aproximadamente 9,3\% de seus quase 10,7 milhões de habitantes tem mais de 65 anos. Isso equivale a um contingente de 162,3 mil idosos (a maioria concentrado em municípios de pequeno e médio porte) (FEE, 2012).

Com o envelhecimento, o perfil nutricional pode sofrer forte influência de fatores ambientais, biológicos e sociais, interferindo na ingestão alimentar (WACHHOLZ; RODRIGUES; YAMANE, 2011). Desta forma, as alterações na mastigação podem causar déficits nutricionais, como também prejudicar a socialização do indivíduo (MEDEIROS; PONTES; MAGALHÃES, 2014).

Um adequado estado de saúde oral aliado a uma boa nutrição são fatores que podem interferir na saúde (PALMER et al., 2010). Com o envelhecimento, os hábitos de mastigação podem mudar. Os idosos são mais propensos a possuir deficiências na função mastigatória e percepção do sabor, em consequência de alterações estruturais, e/ou morfológicas (VAN DER BILT, 2011). Desta forma, as alterações na mastigação podem causar déficits nutricionais, e até mesmo, prejudicar a socialização dos idosos. A presença de uma via oral pobre e/ou debilitada pode gerar impactos na saúde, comprometendo a alimentação, e a nutrição, podendo ser prejudicial à saúde geral do indivíduo (DYE et al., 2007; TRAMONTINO et al., 2009). Para a avaliação das dificuldades encontradas na mastigação, torna-se relevante a autopercepção da saúde oral pelo idoso, durante a alimentação, por meio de administração de questionários, com o intuito de verificar os fatores que possam interferir no desempenho desta função. Assim, diante da necessidade de aprofundar o conhecimento nessa complexa e multidimensional relação, o objetivo deste estudo foi avaliar o estado nutricional, alimentação e a saúde oral de idosos de um município da Serra Gaúcha, no Rio Grande do Sul, Brasil.

\section{Métodos}

Trata-se de um estudo de caráter transversal descritivo, analítico e quantitativo, com a utilização de dados primários. Os dados foram coletados no período entre 2014 e 2015. A amostra foi composta por 135 idosos, sendo 97 mulheres e 38 homens. Conforme cálculo amostral, realizado com auxílio 
do programa Epi Info 3.5.2, estimou-se que, para a prevalência de $29,7 \%$ de desnutrição, conforme (FELIX; SOUZA, 2009), com nível de confiança de 95\%, margem de erro de $8 \%$ e perdas amostrais de $5 \%$, fosse necessária amostra de 132 voluntários.

Os idosos avaliados eram participantes do projeto: Prevenção e Promoção da Saúde da Associação Veranense de Assistência em Saúde (AVAES), no município de Veranópolis, Rio Grande do Sul. Esse estudo foi aprovado pelo Comitê de Ética e Pesquisa da Faculdade Cenecista de Bento Gonçalves sob o no . 796.094. Participaram do estudo os voluntários que assinaram o termo de Consentimento Livre e Esclarecido (TCLE).

As informações obtidas foram coletadas por estudantes de nutrição, sendo todos previamente treinados. As entrevistas e mensuração das variáveis antropométricas levaram em média 30-60 minutos e foram realizadas no Centro Municipal de Convivência da Longevidade São Camilo e/ou coletadas no domicílio.

As variáveis demográficas foram: sexo (feminino e masculino) e idade ( $\geq 60$ anos). As variáveis antropométricas foram: peso ( $\mathrm{kg}$ ), altura (m), índice de massa corporal $\left(\mathrm{IMC}=\mathrm{kg} / \mathrm{m}^{2}\right)$, circunferência da cintura $(\mathrm{CC}=\mathrm{cm})$, circunferência do braço $(\mathrm{CB}=\mathrm{cm})$ e circunferência da panturilha $(\mathrm{CP}=\mathrm{cm})$. O peso corporal foi aferido por balança digital portátil de alta precisão e capacidade de $200 \mathrm{~kg}$, da marca G-Tech ${ }^{\circledast}$. A estatura foi obtida através de fita métrica, com trava, com precisão de $0,1 \mathrm{~cm}$, fixada em parede lisa, sem rodapé e esquadro. Os idosos foram pesados e medidos em pé, descalços, encostando nuca, nádegas e calcanhares na parede, o peso foi distribuído igualmente entre os pés, e a cabeça ficou ereta, com o olhar fixo à frente ou no plano de Frankfurt (MIRANDA et al., 2012).

Classificou-se o Índice de Massa Corporal (IMC), conforme Lipschitz (1994). A circunferência da cintura foi mensurada com fita métrica, com precisão de $0,1 \mathrm{~cm}$, sendo aferida no ponto médio entre a margem costal mais baixa e a crista ilíaca (ROSSI, 2008). Utilizou-se como ponto de corte da circunferência abdominal de acordo com gênero e etnia (SPOSITO, 2007). A circunferência do braço (CB) foi mensurada através de fita métrica posicionada em torno do braço não dominante ou disponível, esta foi medida no ponto médio entre o processo acromial da escápula e o olecrano da ulna (MIRANDA et al., 2012) e classificou-se conforme percentil do NHANES III (1988-1991). A circunferência da panturrilha (CP) foi realizada na perna esquerda, com fita métrica inelástica, na parte mais protuberante. Considerou-se adequada $\mathrm{CP} \geq 31$ (NAJAS, 2005).

Avaliou-se o estado nutricional através das medidas antropométricas e da Mini Avaliação Nutricional (MAN). A MAN foi desenvolvida em 1990 
e permite identificar o risco de desnutrição dos idosos. É composta por 18 tópicos, onde a partir das respostas classificaram-se os idosos em $>23,5$ pontos (bem nutrido), $\geq 17$ pontos a $\leq 23,5$ pontos (risco de desnutrição) e $<17$ pontos (desnutrido) (MIRANDA et al., 2012).

Avaliou-se o consumo alimentar do idoso através do Questionário de Frequência Alimentar (QFA) realizado através do Sistema de Vigilância Alimentar (SISVAN), onde foi possível identificar o consumo habitual de dez grupos alimentares (MIRANDA et al., 2012).

Em relação à saúde bucal, aplicaram-se os questionários GOHAI (Geriatric Oral Health Assessment Index) e EAT-10 (Instrumento de Avaliação da Alimentação). O GOHAI foi criado por Atchison e Dolan (1990) e adaptado para o Brasil por Silva e Castellanos (2001), é composto por doze questões fechadas e com perguntas sobre problemas bucais que afetam funções físicas e funcionais, aspectos psicológicos, dor e desconforto, dos idosos. Cada pergunta apresentava três respostas possíveis: sempre e quase/sempre; algumas vezes e nunca. Elas recebem os escores de 1 a 3, respectivamente, e para determinação do índice, somou-se o escore de cada questão (CARVALHO et al., 2013). Quanto mais alto seu valor, melhor era a autopercepção da saúde oral, escores mais baixos de GOHAI são esperados em indivíduos com piores condições de saúde bucal (ATCHINSON; DOLAN, 1990). Os escores finais foram classificados como alto (34 a 36), moderado (31 a 33) e baixo (menor que 30) (SILVA et al., 2005).

O Instrumento de Avaliação da Alimentação (EAT-10), é uma ferramenta para avaliação do risco de disfagia, auxilia na indicação de intervenção precoce. É constituído por dez questões de formulação simples, fornecendo informações sobre funcionalidade, impacto emocional e sintomas físicos. Possuía uma escala de 0 a 4 , onde a pontuação $\geq 3$ era indicativo de anormalidade de deglutição (BELAFSKY et al., 2008).

A análise dos dados foi realizada utilizando o programa estatístico Statistical Package for Social Sciences (SPSS ${ }^{\circledR}$ ) versão 21.0 e foi realizada conforme as características dos dados. As variáveis quantitativas foram descritas por média e desvio padrão, e as categóricas por frequências absolutas e relativas. Para comparar médias entre os grupos, o teste t-student para amostras independentes foi aplicado. Na comparação de proporções, os testes qui-quadrado de Pearson ou exato de Fisher foram utilizados. Em caso de significância estatística, a análise dos resíduos ajustadas foi aplicada. Considerou-se o nível de significância de $5 \%(p<0,05)$. 


\section{Resultados}

Do total de 135 idosos avaliados, 71,9\% (97) eram mulheres. A idade média foi de 73,4 $\pm 7,2$ (mínimo=60 anos e máximo=93 anos). Ao realizar a distribuição das faixas etárias encontrou-se que 31,1\% (42) estavam na faixa etária de 60-69 anos, 46,7\% (63) em 70-79 anos, 20\% (27) em 80-89 anos e apenas 2,2\% (3) possuíam idade $\geq 90$ anos.

Conforme Tabela 1 , na comparação do estado nutricional entre homens e mulheres, a obesidade abdominal foi mais prevalente nas mulheres $(\mathrm{p}=0,037)$.

Tabela 1 - Estado nutricional através da avaliação do IMC, CC, CB, CP e da MAN em idosos conforme gênero.

\begin{tabular}{|c|c|c|c|c|}
\hline Características & $\begin{array}{c}\text { Amostra } \\
\text { total } \\
\mathrm{n}=135 \\
\mathrm{n}(\%)\end{array}$ & $\begin{array}{c}\text { Homens } \\
n=38 \\
n(\%)\end{array}$ & $\begin{array}{c}\text { Mulheres } \\
n=97 \\
n(\%)\end{array}$ & $\mathrm{p}$ \\
\hline IMC $\left(\mathrm{kg} / \mathrm{m}^{2}\right)-$ média $\pm \mathrm{DP}$ & $28,3 \pm 5,3$ & $27,7 \pm 4,6$ & $28,5 \pm 5,5$ & 0,402 \\
\hline Baixo peso $(<22$ kg/m²) & $12(8,9)$ & $3(7,9)$ & $9(9,3)$ & \\
\hline Eutrofia ( $\geq 22$ a $\leq 27$ kg/m²) & $47(34,8)$ & $16(42,1)$ & $31(32,0)$ & 0,538 \\
\hline Sobrepeso (>27 kg/m²) & $76(56,3)$ & $19(50,0)$ & $57(58,8)$ & \\
\hline $\mathrm{CC}(\mathrm{cm})-$ média $\pm \mathrm{DP}$ & $100 \pm 13,0$ & $105 \pm 10,1$ & $98,1 \pm 13,5$ & 0,004 \\
\hline Normal & $44(32,6)$ & $18(47,4)$ & $26(26,8)$ & 0,037 \\
\hline Obesidade abdominal & $91(67,4)$ & $20(52,6)$ & $71(73,2)$ & \\
\hline $\mathrm{CB}-$ média $\pm \mathrm{DP}$ & $31,2 \pm 3,8$ & $31,2 \pm 3,6$ & $31,2 \pm 3,9$ & 0,982 \\
\hline Obesidade & $11(8,1)$ & $4(10,5)$ & $7(7,2)$ & \\
\hline Sobrepeso & $3(2,2)$ & $\mathrm{O}(0,0)$ & $3(3,1)$ & \\
\hline Risco de sobrepeso & $15(11,1)$ & $2(5,3)$ & $13(13,4)$ & 0,207 \\
\hline Eutrofia & $82(60,7)$ & $21(55,3)$ & $61(62,9)$ & \\
\hline Risco nutricional & $17(12,6)$ & $8(21,1)$ & $9(9,3)$ & \\
\hline Desnutrição & $7(5,2)$ & $3(7,9)$ & $4(4,1)$ & \\
\hline $\mathrm{CP}-$ média $\pm \mathrm{DP}$ & $37,0 \pm 3,9$ & $37,3 \pm 3,9$ & $36,9 \pm 4,0$ & 0,576 \\
\hline Risco nutricional $(<31)$ & $3(2,2)$ & $1(2,6)$ & $2(2,1)$ & 1,000 \\
\hline Adequado ( $\geq 31$ ) & $132(97,8)$ & $37(97,4)$ & $95(97,9)$ & \\
\hline
\end{tabular}

Continua 


\begin{tabular}{l|l|l|l|l}
\hline \multicolumn{1}{c|}{ Características } & \multicolumn{1}{|c|}{$\begin{array}{c}\text { Amostra } \\
\text { total } \\
\mathrm{n}=135 \\
\mathrm{n}(\%)\end{array}$} & $\begin{array}{c}\text { Homens } \\
\mathrm{n}=38 \\
\mathrm{n}(\%)\end{array}$ & $\begin{array}{c}\text { Mulheres } \\
\mathrm{n}=97 \\
\mathrm{n}(\%)\end{array}$ & $\mathrm{p}$ \\
\hline $\begin{array}{l}\text { MAN (pontuação total) - } \\
\text { média } \pm \text { DP }\end{array}$ & $27,3 \pm 2,2$ & $27,5 \pm 2,2$ & $27,2 \pm 2,1$ & 0,488 \\
\hline Bem nutrido & $119(88,1)$ & $34(89,5)$ & $85(87,6)$ & \\
\hline Risco de desnutrição & $15(11,1)$ & $4(10,5)$ & $11(11,3)$ & 0,811 \\
\hline Desnutrido & $1(0,7)$ & $0(0,0)$ & $1(1,0)$ & \\
\hline
\end{tabular}

Legenda: $n=$ número; \%=porcentagem; * $p<0,05$; IMC=índice de massa corporal;

$\mathrm{CC}=$ Circunferência da cintura; $\mathrm{CB}=$ circunferência do braço; $\mathrm{CP}=$ circunferência da panturrilha;

MAN=Mini avalição nutricional; * associação estatisticamente significativa pelo teste dos resíduos ajustados a 5\% de significância.

Fonte: Tabela elaborada pelos autores.

E comparando o estado nutricional entre as faixas etárias, tanto o risco de desnutrição pela MAN quanto à prevalência de baixo peso pelo IMC aumentaram com o avanço da idade, principalmente no grupo de 80 anos ou mais ( $p=0,021$ e $p=0,039$, respectivamente), conforme a Figura 1 (a) e (b). Para os demais métodos de avaliação nutricional, as diferenças não foram estatisticamente significativas $(\mathrm{p}>0,05)$. 
Figura 1 - Associação da classificação da Mini Avalição Nutricional (MAN) (a) e da classificação do Índice de Massa Corporal (IMC) (b) conforme faixa etária.

(a)

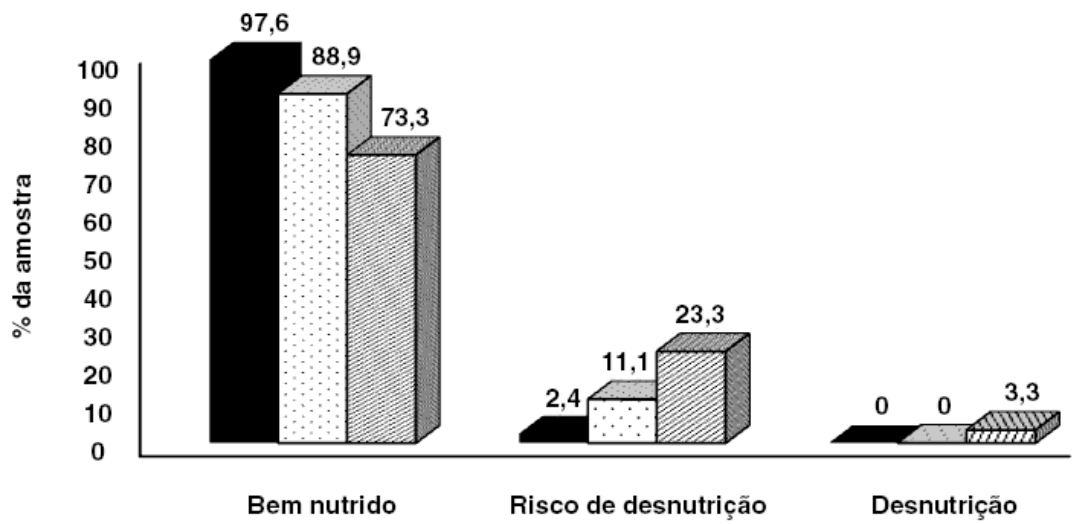

Classificação MAN

- 60-69 anos $\square 70-79$ anos $\$ 80$ anos ou mais

Fonte: Figura elaborada pelos autores.

(b)

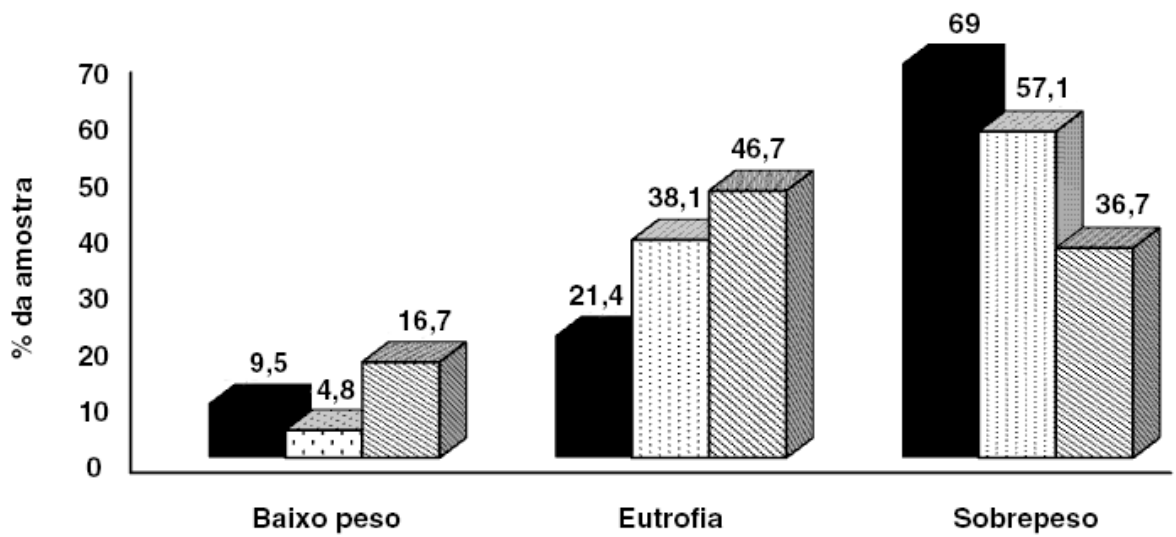

Classificação do IMC

- $60-69$ anos $\square 70-79$ anos 780 anos ou mais

Fonte: Figura elaborada pelos autores. 
Quando foram analisados os parâmetros da MAN entre os gêneros, houve diferença significativa somente na comparação da saúde com as pessoas de mesma idade. As mulheres se perceberam com melhor saúde quando comparadas aos homens, conforme apresenta a Tabela 2.

Tabela 2 - Avaliação da MAN entre os idosos, conforme gênero.

\begin{tabular}{|c|c|c|c|c|}
\hline Característica & $\begin{array}{c}\text { Amostra } \\
\text { total } \\
\mathrm{n}=135 \\
\mathrm{n}(\%)\end{array}$ & $\begin{array}{c}\text { Homens } \\
n=38 \\
n(\%)\end{array}$ & $\begin{array}{c}\text { Mulheres } \\
\text { n=97 } \\
\mathrm{n}(\%)\end{array}$ & $\mathrm{p}$ \\
\hline \multicolumn{4}{|l|}{ Diminuição na ingestão } & 0,509 \\
\hline Diminuição severa & $4(3,0)$ & $1(2,6)$ & $3(3,1)$ & \\
\hline Diminuição moderada & $34(25,2)$ & $7(18,4)$ & $27(27,8)$ & \\
\hline Sem diminuição & $97(71,9)$ & $30(78,9)$ & $67(69,1)$ & \\
\hline \multicolumn{4}{|l|}{ Perda de peso } & 0,193 \\
\hline Superior a 3kg & $7(5,2)$ & $3(7,9)$ & $4(4,1)$ & \\
\hline Não sabe informar & $8(5,9)$ & $0(0,0)$ & $8(8,2)$ & \\
\hline Entre 1 e $3 \mathrm{~kg}$ & $23(17,0)$ & $5(13,2)$ & $18(18,6)$ & \\
\hline Sem perda de peso & $97(71,9)$ & $30(78,9)$ & $67(69,1)$ & \\
\hline \multicolumn{4}{|l|}{ Mobilidade } & 1,000 \\
\hline $\begin{array}{l}\text { Deambula, mas não é } \\
\text { capaz de sair de casa }\end{array}$ & $2(1,5)$ & $0(0,0)$ & $2(2,1)$ & \\
\hline Normal & $133(98,5)$ & $38(100)$ & $95(97,9)$ & \\
\hline \multicolumn{4}{|l|}{$\begin{array}{l}\text { Estresse psicológico } \\
\text { ou doença aguda }\end{array}$} & 0,350 \\
\hline $\operatorname{Sim}$ & $38(28,1)$ & $8(21,1)$ & $30(30,9)$ & \\
\hline Não & $97(71,9)$ & $30(78,9)$ & $67(69,1)$ & \\
\hline \multicolumn{4}{|l|}{$\begin{array}{l}\text { Problemas } \\
\text { neuropsicológicos }\end{array}$} & 0,772 \\
\hline Demência leve & $16(11,9)$ & $5(13,2)$ & $11(11,3)$ & \\
\hline Sem problemas & $119(88,1)$ & $33(86,8)$ & $86(88,7)$ & \\
\hline \multicolumn{4}{|l|}{ Classificação do IMC } & 0,558 \\
\hline$<19 \mathrm{~kg} / \mathrm{m} 2$ & $2(1,5)$ & $\mathrm{O}(0,0)$ & $2(2,1)$ & \\
\hline
\end{tabular}

Continua 


\begin{tabular}{|c|c|c|c|c|}
\hline Característica & $\begin{array}{c}\text { Amostra } \\
\text { total } \\
\mathrm{n}=135 \\
\mathrm{n}(\%)\end{array}$ & $\begin{array}{l}\text { Homens } \\
n=38 \\
n(\%)\end{array}$ & $\begin{array}{l}\text { Mulheres } \\
\qquad \mathrm{n}=97 \\
\mathrm{n}(\%)\end{array}$ & $\mathrm{p}$ \\
\hline$\geq 19 \mathrm{~kg} / \mathrm{m} 2$ e $<21 \mathrm{~kg} / \mathrm{m} 2$ & $6(4,4)$ & $2(5,3)$ & $4(4,1)$ & \\
\hline$\geq 21 \mathrm{~kg} / \mathrm{m} 2$ e $<23 \mathrm{~kg} / \mathrm{m} 2$ & $13(9,6)$ & $2(5,3)$ & $11(11,3)$ & \\
\hline$\geq 23 \mathrm{~kg} / \mathrm{m} 2$ & $114(84,4)$ & $34(89,5)$ & $80(82,5)$ & \\
\hline \multicolumn{4}{|l|}{$\begin{array}{l}\text { Paciente vive em seu } \\
\text { domicílio }\end{array}$} & 0,620 \\
\hline Sim & $130(96,3)$ & $36(94,7)$ & $94(96,9)$ & \\
\hline Não & $5(3,7)$ & $2(5,3)$ & $3(3,1)$ & \\
\hline \multicolumn{4}{|l|}{$\begin{array}{l}\text { Utiliza mais de } 3 \\
\text { medicamentos }\end{array}$} & 0,051 \\
\hline Sim & $80(59,3)$ & $17(44,7)$ & $63(64,9)$ & \\
\hline Não & $55(40,7)$ & $21(55,3)$ & $34(35,1)$ & \\
\hline \multicolumn{4}{|l|}{ Lesões da pele } & 0,249 \\
\hline Sim & $17(12,6)$ & $7(18,4)$ & $10(10,3)$ & \\
\hline Não & $118(87,4)$ & $31(81,6)$ & $87(89,7)$ & \\
\hline \multicolumn{4}{|l|}{$\begin{array}{l}\text { Quantidade de } \\
\text { refeições/dia }\end{array}$} & 0,227 \\
\hline Uma & $1(0,7)$ & $\mathrm{O}(0,0)$ & $1(1,0)$ & \\
\hline Duas & $5(3,7)$ & $3(7,9)$ & $2(2,1)$ & \\
\hline Três & $129(95,6)$ & $35(92,1)$ & $94(96,9)$ & \\
\hline \multicolumn{4}{|l|}{$\begin{array}{l}\text { Consome } 1 \text { porção diária } \\
\text { de leite ou derivados }\end{array}$} & 0,361 \\
\hline Sim & $120(88,9)$ & $32(84,2)$ & $88(90,7)$ & \\
\hline Não & $15(11,1)$ & $6(15,8)$ & $9(9,3)$ & \\
\hline \multicolumn{4}{|l|}{$\begin{array}{l}\text { Consome } 2 \text { porções ou + } \\
\text { semanais de legumes } \\
\text { ou ovos }\end{array}$} & 0,053 \\
\hline $\operatorname{Sim}$ & $129(95,6)$ & $34(89,5)$ & $95(97,9)$ & \\
\hline Não & $6(4,4)$ & $4(10,5)$ & $2(2,1)$ & \\
\hline
\end{tabular}

Continua 


\begin{tabular}{|c|c|c|c|c|}
\hline Característica & $\begin{array}{c}\text { Amostra } \\
\text { total } \\
\mathrm{n}=135 \\
\mathrm{n}(\%)\end{array}$ & $\begin{array}{l}\text { Homens } \\
\qquad n=38 \\
n(\%)\end{array}$ & $\begin{array}{l}\text { Mulheres } \\
\qquad n=97 \\
n(\%)\end{array}$ & $p$ \\
\hline \multicolumn{4}{|l|}{$\begin{array}{l}\text { Consome: carnes, peixes } \\
\text { ou aves todos os dias }\end{array}$} & 0,397 \\
\hline $\operatorname{Sim}$ & $84(62,2)$ & $21(55,3)$ & $63(64,9)$ & \\
\hline Não & $51(37,8)$ & $17(44,7)$ & $34(35,1)$ & \\
\hline \multicolumn{4}{|l|}{$\begin{array}{l}\text { Consome } 2 \text { porções } \\
\text { ou + diárias de frutas } \\
\text { ou vegetais }\end{array}$} & 0,291 \\
\hline $\operatorname{Sim}$ & $11(8,1)$ & $5(13,2)$ & $6(6,2)$ & \\
\hline Não & $124(91,9)$ & $33(86,8)$ & $91(93,8)$ & \\
\hline \multicolumn{4}{|l|}{$\begin{array}{l}\text { Consome: quantidade } \\
\text { de copos de líquido por dia }\end{array}$} & 0,256 \\
\hline$<3$ copos & $25(18,5)$ & $8(21,1)$ & $17(17,5)$ & \\
\hline 3 a 5 copos & $54(40,0)$ & $11(28,9)$ & $43(44,3)$ & \\
\hline$>5$ copos & $56(41,5)$ & $19(50,0)$ & $37(38,1)$ & \\
\hline \multicolumn{4}{|l|}{ Modo de se alimentar } & 0,281 \\
\hline $\begin{array}{l}\text { Alimenta-se sozinho com } \\
\text { dificuldade }\end{array}$ & $1(0,7)$ & $1(2,6)$ & $0(0,0)$ & \\
\hline Alimenta-se sozinho & $134(99,3)$ & $37(97,4)$ & $97(100)$ & \\
\hline \multicolumn{4}{|l|}{ Problema nutricional } & 0,215 \\
\hline Acredita estar desnutrido & $2(1,5)$ & $1(2,6)$ & $1(1,0)$ & \\
\hline Não sabe dizer & $1(0,7)$ & $1(2,6)$ & $0(0,0)$ & \\
\hline Acredita não ter problema & $132(97,8)$ & $36(94,7)$ & $96(99,0)$ & \\
\hline \multicolumn{4}{|l|}{$\begin{array}{l}\text { Comparação da saúde } \\
\text { com as pessoas de } \\
\text { mesma idade }\end{array}$} & 0,030 \\
\hline Não muito boa & $8(5,9)$ & $4(10,5)$ & $4(4,1)$ & \\
\hline Não sabe informar & $10(7,4)$ & $5(13,2)$ & $5(5,2)$ & \\
\hline Boa & $105(77,8)$ & $29(76,3)$ & $76(78,4)$ & \\
\hline Melhor & $12(8,9)$ & $\mathrm{O}(0,0)$ & $12(12,4)^{*}$ & \\
\hline
\end{tabular}




\begin{tabular}{|c|c|c|c|c|}
\hline Característica & $\begin{array}{c}\text { Amostra } \\
\text { total } \\
\mathrm{n}=135 \\
\mathrm{n}(\%)\end{array}$ & $\begin{array}{c}\text { Homens } \\
n=38 \\
n(\%)\end{array}$ & $\begin{array}{c}\text { Mulheres } \\
\mathrm{n}=97 \\
\mathrm{n}(\%)\end{array}$ & $p$ \\
\hline \multicolumn{5}{|c|}{$\begin{array}{l}\text { Classificação Circunferên- } \\
\text { cia do Braço }\end{array}$} \\
\hline$>22 \mathrm{~cm}$ & $135(100)$ & $38(100)$ & $97(100)$ & - \\
\hline \multicolumn{5}{|c|}{$\begin{array}{l}\text { Classificação Circunferên- } \\
\text { cia da Panturrilha }\end{array}$} \\
\hline$<31 \mathrm{~cm}$ & $3(2,2)$ & $1(2,6)$ & $2(2,1)$ & 1,000 \\
\hline$\geq 31 \mathrm{~cm}$ & $132(97,8)$ & $37(97,4)$ & $95(97,9)$ & \\
\hline
\end{tabular}

Legenda MAN: n=número; \%=porcentagem; p<0,05; IMC=índice de massa corporal * associação estatisticamente significativa pelo teste dos resíduos ajustados a 5\% de significância.

Fonte: Tabela elaborada pelos autores.

Ao investigar a saúde oral, através dos instrumentos EAT-10 e GOHAI, observamos que não houve diferença significativa entre os gêneros ( $p>0,05)$, conforme pode ser visualizado na Tabela 3 . Apenas $4,4 \%$ apresentavam problema de deglutição conforme o EAT-10. Através do GOHAI, verificou-se que apenas $17,8 \%$ se classificaram com baixa percepção da saúde oral.

Tabela 3 - Saúde oral (mastigação e deglutição) através dos instrumentos EAT-10 e GOHAl em idosos, conforme o gênero.

\begin{tabular}{l|l|l|l|l}
\hline \multicolumn{1}{c|}{ Característica } & $\begin{array}{c}\text { Amostra } \\
\text { total } \\
\mathbf{n = 1 3 5} \\
\mathbf{n}(\%)\end{array}$ & $\begin{array}{c}\text { Homens } \\
\mathbf{n}=38 \\
\mathbf{n}(\%)\end{array}$ & $\begin{array}{c}\text { Mulheres } \\
\mathbf{n}=97 \\
\mathbf{n}(\%)\end{array}$ & p \\
\hline $\begin{array}{l}\text { Instrumento de avaliação } \\
\text { da alimentação - EAT 10 }\end{array}$ & $129(95,6)$ & $38(100)$ & $91(93,8)$ & \\
\hline $\begin{array}{l}\text { Sem problemas } \\
\text { Problemas de deglutição } \\
\text { (>3 pontos) }\end{array}$ & $6(4,4)$ & $0(0,0)$ & $6(6,2)$ & \\
\hline
\end{tabular}

Continua 


\begin{tabular}{|c|c|c|c|c|}
\hline \multicolumn{4}{|l|}{$\begin{array}{l}\text { GOHAI (Geriatric Oral } \\
\text { Health Assessment Index) }\end{array}$} & \multirow[t]{2}{*}{0,454} \\
\hline $\begin{array}{l}\text { Alta percepção (34 a } 36 \\
\text { pontos) }\end{array}$ & $80(59,3)$ & $25(65,8)$ & $55(56,7)$ & \\
\hline $\begin{array}{l}\text { Moderada (31 a } 33 \\
\text { pontos) }\end{array}$ & $31(23,0)$ & $6(15,8)$ & $25(25,8)$ & \\
\hline Baixa ( $\leq 30$ pontos) & $24(17,8)$ & $7(18,4)$ & $17(17,5)$ & \\
\hline
\end{tabular}

Legenda: n=número; \%=porcentagem; * $\mathrm{p}<0,05$; EAT 10=Instrumento de Avaliação da Alimentação; $\mathrm{GOHAl}=$ Geriatric Oral Health Assessment Index, * associação estatisticamente significativa pelo teste dos resíduos ajustados a 5\% de significância.

Fonte: Tabela elaborada pelos autores.

Na avaliação do consumo alimentar entre os gêneros, houve diferença quanto ao consumo de frutas frescas $(p=0,006)$ e batata/salgados fritos $(p=0,001)$. Observou-se que o consumo de frutas frescas foi maior nas mulheres e batata/ salgados fritos foi mais consumido entre os homens, conforme apresenta a Tabela 4.

Tabela 4 - Avaliação do questionário de frequência alimentar em idosos, segundo gênero.

\begin{tabular}{|c|c|c|c|c|}
\hline Consumo semanal & $\begin{array}{c}\text { Amostra } \\
\text { total } \\
n=135 \\
n(\%)\end{array}$ & $\begin{array}{c}\text { Homens } \\
n=38 \\
n(\%)\end{array}$ & $\begin{array}{c}\text { Mulheres } \\
\mathrm{n}=97 \\
\mathrm{n}(\%)\end{array}$ & $\mathrm{p}$ \\
\hline \multicolumn{4}{|l|}{$\begin{array}{l}\text { Legumes de verduras } \\
\text { cozidos }\end{array}$} & 0,465 \\
\hline 0 & $8(5,9)$ & $3(7,9)$ & $5(5,2)$ & \\
\hline 1 & $13(9,6)$ & $2(5,3)$ & $11(11,3)$ & \\
\hline 2 & $23(17,0)$ & $8(21,1)$ & $15(15,5)$ & \\
\hline 3 & $12(8,9)$ & $4(10,5)$ & $8(8,2)$ & \\
\hline 4 & $7(5,2)$ & $1(2,6)$ & $6(6,2)$ & \\
\hline 5 & $12(8,9)$ & $3(7,9)$ & $9(9,3)$ & \\
\hline 6 & $9(6,7)$ & $5(13,2)$ & $4(4,1)$ & \\
\hline 7 & $51(37,8)$ & $12(31,6)$ & $39(40,2)$ & \\
\hline
\end{tabular}

Continua 


\begin{tabular}{|c|c|c|c|c|}
\hline Consumo semanal & $\begin{array}{c}\text { Amostra } \\
\text { total } \\
n=135 \\
n(\%)\end{array}$ & $\begin{array}{c}\text { Homens } \\
n=38 \\
n(\%)\end{array}$ & $\begin{array}{c}\text { Mulheres } \\
\mathrm{n}=97 \\
\mathrm{n}(\%)\end{array}$ & $\mathrm{p}$ \\
\hline \multicolumn{4}{|l|}{ Frutas frescas } & 0,006 \\
\hline 1 & $2(1,5)$ & $0(0,0)$ & $2(2,1)$ & \\
\hline 2 & $2(1,5)$ & $2(5,3)^{\star}$ & $0(0,0)$ & \\
\hline 3 & $3(2,2)$ & $3(7,9)^{\star}$ & $\mathrm{O}(0,0)$ & \\
\hline 4 & $3(2,2)$ & $1(2,6)$ & $2(2,1)$ & \\
\hline 5 & $5(3,7)$ & $3(7,9)$ & $2(2,1)$ & \\
\hline 6 & $4(3,0)$ & $0(0,0)$ & $4(4,1)$ & \\
\hline 7 & $116(85,9)$ & $29(76,3)$ & $87(89,7)^{\star}$ & \\
\hline \multicolumn{4}{|l|}{ Feijão } & 0,932 \\
\hline 0 & $17(12,6)$ & $4(10,5)$ & $13(13,4)$ & \\
\hline 1 & $42(31,1)$ & $10(26,3)$ & $32(33,0)$ & \\
\hline 2 & $34(25,2)$ & $9(23,7)$ & $25(25,8)$ & \\
\hline 3 & $16(11,9)$ & $5(13,2)$ & $11(11,3)$ & \\
\hline 4 & $6(4,4)$ & $2(5,3)$ & $4(4,1)$ & \\
\hline 5 & $5(3,7)$ & $2(5,3)$ & $3(3,1)$ & \\
\hline 6 & $4(3,0)$ & $2(5,3)$ & $2(2,1)$ & \\
\hline 7 & $11(8,1)$ & $4(10,5)$ & $7(7,2)$ & \\
\hline \multicolumn{4}{|l|}{ Leite ou iogurte } & 0,226 \\
\hline 0 & $18(13,3)$ & $6(15,8)$ & $12(12,4)$ & \\
\hline 3 & $1(0,7)$ & $1(2,6)$ & $\mathrm{O}(0,0)$ & \\
\hline 4 & $1(0,7)$ & $0(0,0)$ & $1(1,0)$ & \\
\hline 5 & $3(2,2)$ & $2(5,3)$ & $1(1,0)$ & \\
\hline 7 & $112(83,0)$ & $29(76,3)$ & $83(85,6)$ & \\
\hline \multicolumn{4}{|l|}{ Batata frita, salgados fritos } & 0,001 \\
\hline 0 & $82(60,7)$ & $22(57,9)$ & $60(61,9)$ & \\
\hline 1 & $29(21,5)$ & $3(7,9)$ & $26(26,8)^{\star}$ & \\
\hline 2 & $18(13,3)$ & $8(21,1)$ & $10(10,3)$ & \\
\hline 3 & $4(3,0)$ & $4(10,5)^{\star}$ & $\mathrm{O}(0,0)$ & \\
\hline 5 & $1(0,7)$ & $0(0,0)$ & $1(1,0)$ & \\
\hline 7 & $1(0,7)$ & $1(2,6)$ & $\mathrm{O}(0,0)$ & \\
\hline
\end{tabular}

Continua 


\begin{tabular}{|c|c|c|c|c|}
\hline Consumo semanal & $\begin{array}{c}\text { Amostra } \\
\text { total } \\
\mathrm{n}=135 \\
\mathrm{n}(\%)\end{array}$ & $\begin{array}{c}\text { Homens } \\
n=38 \\
n(\%)\end{array}$ & $\begin{array}{c}\text { Mulheres } \\
\begin{array}{c}n=97 \\
n(\%)\end{array}\end{array}$ & $p$ \\
\hline \multicolumn{4}{|l|}{ Hambúrguer e embutidos } & 0,060 \\
\hline 0 & $57(42,2)$ & $9(23,7)$ & $48(49,5)$ & \\
\hline 1 & $23(17,0)$ & $8(21,1)$ & $15(15,5)$ & \\
\hline 2 & $23(17,0)$ & $7(18,4)$ & $16(16,5)$ & \\
\hline 3 & $9(6,7)$ & $4(10,5)$ & $5(5,2)$ & \\
\hline 4 & $5(3,7)$ & $1(2,6)$ & $4(4,1)$ & \\
\hline 5 & $8(5,9)$ & $5(13,2)$ & $3(3,1)$ & \\
\hline 6 & $2(1,5)$ & $0(0,0)$ & $2(2,1)$ & \\
\hline 7 & $8(5,9)$ & $4(10,5)$ & $4(4,1)$ & \\
\hline \multicolumn{4}{|l|}{$\begin{array}{l}\text { Bolachas/biscoitos } \\
\text { salgados }\end{array}$} & 0,459 \\
\hline 0 & $32(23,7)$ & $10(26,3)$ & $22(22,7)$ & \\
\hline 1 & $12(8,9)$ & $4(10,5)$ & $8(8,2)$ & \\
\hline 2 & $12(8,9)$ & $6(15,8)$ & $6(6,2)$ & \\
\hline 3 & $15(11,1)$ & $3(7,9)$ & $12(12,4)$ & \\
\hline 4 & $12(8,9)$ & $2(5,3)$ & $10(10,3)$ & \\
\hline 5 & $16(11,9)$ & $2(5,3)$ & $14(14,4)$ & \\
\hline 6 & $5(3,7)$ & $2(5,3)$ & $3(3,1)$ & \\
\hline 7 & $31(23,0)$ & $9(23,7)$ & $22(22,7)$ & \\
\hline \multicolumn{4}{|l|}{ Bolachas/biscoitos doces } & 0,229 \\
\hline 0 & $33(24,4)$ & $9(23,7)$ & $24(24,7)$ & \\
\hline 1 & $19(14,1)$ & $9(23,7)$ & $10(10,3)$ & \\
\hline 2 & $24(17,8)$ & $5(13,2)$ & $19(19,6)$ & \\
\hline 3 & $15(11,1)$ & $3(7,9)$ & $12(12,4)$ & \\
\hline 4 & $11(8,1)$ & $2(5,3)$ & $9(9,3)$ & \\
\hline 5 & $13(9,6)$ & $2(5,3)$ & $11(11,3)$ & \\
\hline 6 & $2(1,5)$ & $\mathrm{O}(0,0)$ & $2(2,1)$ & \\
\hline 7 & $18(13,3)$ & $8(21,1)$ & $10(10,3)$ & \\
\hline \multicolumn{4}{|l|}{ Refrigerante } & 0,699 \\
\hline 0 & $79(58,5)$ & $22(57,9)$ & $57(58,8)$ & \\
\hline 1 & $25(18,5)$ & $6(15,8)$ & $19(19,6)$ & \\
\hline
\end{tabular}

Continua 


\begin{tabular}{l|l|l|l|l}
\hline Consumo semanal & $\begin{array}{c}\text { Amostra } \\
\text { total } \\
\mathbf{n = 1 3 5} \\
\mathbf{n}(\%)\end{array}$ & $\begin{array}{c}\text { Homens } \\
\mathbf{n = 3 8} \\
\mathbf{n}(\%)\end{array}$ & $\begin{array}{c}\text { Mulheres } \\
\mathbf{n}=\mathbf{9 7} \\
\mathbf{n}(\%)\end{array}$ & $\mathbf{p}$ \\
\hline 2 & $18(13,3)$ & $6(15,8)$ & $12(12,4)$ & \\
\hline 3 & $3(2,2)$ & $1(2,6)$ & $2(2,1)$ & \\
\hline 5 & $2(1,5)$ & $0(0,0)$ & $2(2,1)$ & \\
\hline 6 & $1(0,7)$ & $1(2,6)$ & $0(0,0)$ & \\
\hline 7 & $7(5,2)$ & $2(5,3)$ & $5(5,2)$ & \\
\hline
\end{tabular}

Legenda: $n=$ número; \%=porcentagem; * $\mathrm{p}<0,005$. * associação estatisticamente significativa pelo teste dos resíduos ajustados a 5\% de significância.

Fonte: Tabela elaborada pelos autores.

Não houve associação significativa entre os resultados da saúde oral com o consumo alimentar $(\mathrm{p}>0,05)$.

\section{Discussão}

A maioria da amostra estudada foi composta por mulheres. O predomínio de idosas em pesquisas voltadas para a terceira idade pode ser observado em outros estudos, conforme cita Nascimento et al. (2011). Além disso, observa-se uma feminização do envelhecimento, onde de acordo com IBGE (2017), no Mato Grosso do Sul as mulheres nascidas em 2015 têm expectativa de viver 7,1 anos mais que os homens. Enquanto os do gênero masculino devem chegar aos 71,9 anos as mulheres chegarão aos 79 anos. Em outro estudo feito por Camargos e Gonzaga (2015), as mulheres apresentaram maiores expectativas de vida, quando comparadas aos homens. Perelman, Fernandes e Mateus. (2012) demonstraram que as mulheres vivem mais. As principais explicações para este paradoxo são que as mulheres sofrem mais de doenças e adotam atitudes diferentes em relação ao cuidado com a saúde. E no presente estudo, os idosos vivem em um município pequeno como é Veranópolis, onde a maioria das idosas trabalha no lar, com atividades laborais como a horta, culinária para a família, se expondo menos às situações de periculosidade e dedicando-se mais ao cuidado com a família.

Dentre os avaliados, a minoria dos idosos possuía idade $\geq 90$ anos, este fato pode ser atribuído ao aumento da expectativa de vida em todos os municípios brasileiros, e também por Veranópolis, RS apresentar expectativa média de vida ao nascer similar as observadas em países desenvolvidos e industrializados 
(CRUZ; MORIGUCHI, 2002). Ainda, de acordo com dados do Atlas do Desenvolvimento Humano do Brasil (ATLAS BRASIL, 2013), a esperança de vida ao nascer da população veranense teve aumento de 3,7 anos entre 1991 e 2010, chegando a 75,3 anos.

Outro resultado encontrado no presente estudo, é que a maioria das mulheres possuía obesidade abdominal (73,2\%). Trabalho realizado em Porto Alegre, Rio Grande do Sul, demonstrou que a prevalência de obesidade abdominal nessa população foi de 30,6\%, sendo maior nas mulheres (VENTURINI et al., 2013). Bassler e Lei (2008) em seu estudo realizado na região metropolitana de Curitiba mostraram que $57 \%$ dos idosos estavam acima do peso, sendo a maior frequência (61\%) encontrada nas mulheres. O sobrepeso na maioria das vezes está relacionado ao gênero feminino, visto que as mulheres têm maior chance de apresentarem sobrepeso ou obesidade pelo maior acúmulo de gordura visceral e alterações hormonais comuns na menopausa acompanhada por aumento de peso e adiposidade (CAMPOS et al., 2006).

Ao comparar o estado nutricional entre as faixas etárias, tanto o risco de desnutrição pelo MAN quanto à prevalência de baixo peso pelo IMC aumentaram com o avanço da idade. Em um estudo realizado por Morais et al. (2010) objetivando diagnosticar o estado nutricional de idosos através da associação entre o IMC e a MAN observou-se que dos $34 \%$ de indivíduos estavam com sobrepeso pelo IMC, 6\% deles estavam desnutridos e $15 \%$ em risco de desnutrição segundo a MAN. A alta prevalência de desnutrição em idosos, avaliados por meio da MAN, também foi mostrada por Sperotto e Spinelli (2010), em que $35 \%$ estavam desnutridos e $65 \%$ com risco de desnutrição.

Através da MAN, observou-se que as mulheres se percebem com melhor saúde quando comparadas aos homens. Braz (2005), concluiu, a partir dos seus dados, que houve desfavorecimento significativo em termos de saúde em relação aos homens. Eles morrem mais cedo do que as mulheres e, na maioria das vezes, recorrem menos às consultas. Em outra publicação realizada por Gomes et al. (2007), reforçaram, a ideia da menor procura por serviços de saúde por parte de homens.

Quando se analisou a saúde oral pelos indicadores GOHAI e EAT-10 não houve dados significativos ( $p>0,05$ ). Estudo realizado com idosos apontou que a autopercepção se relacionou significativamente com a capacidade funcional, mostrando que os idosos que levavam uma vida mais independente tinham melhor percepção sobre sua mastigação (MORYA et al., 2012). Silva e Castellanos (2001) encontraram um resultado através do GOHAI de 33,80\% para indivíduos dentados. Pelo GOHAI, na nossa amostra, 59,3\% dos idosos 
tiveram escores classificados como altos, demonstrando um resultado positivo para a saúde oral e superior aos citados acima.

Lewandowski e Bós (2014), em um estudo com idosos longevos obtiveram resultados contrários aos desta pesquisa, a saúde bucal dos longevos segundo o GOHAI, foi considerada ruim pela maioria (60,6\%). Para Nunes e Abegg (2008), os idosos percebem a sua saúde bucal fazendo uso, de sinais e sintomas como dor, ou de problemas que afetam a mastigação e interferem na aparência. Além disso, os autores referem que, a percepção da saúde bucal pode ser influenciada por crenças e pelo valor atribuído à saúde perdida pelo indivíduo afetado. A utilização desse índice demonstrou ser um importante preditor para indicar problemas bucais. Além disso, trata-se de um instrumento que pode ser aplicado por profissionais da área da saúde e pode ser utilizado como um meio de identificar a necessidade de encaminhamento para a avaliação odontológica pelos profissionais odontólogos (LEWANDOWSKI; BÓS, 2014).

Em relação ao EAT-10, 95,6\% dos idosos avaliados declararam não ter problemas de deglutição; isso pode ser atribuído ao fato dos idosos avaliados pertencerem a um grupo ativo, como é o caso do projeto de prevenção em Veranópolis. Cassol et al. (2012) concluíram que idosos saudáveis, normalmente, não auto-referem alterações significativas quanto à qualidade de vida em deglutição. Além disso, o uso de prótese dentária, quando bem adaptada, não causa prejuízos na qualidade de vida tanto na mastigação quanto na deglutição. Rofes et al. (2014), tiveram por objetivo avaliar a acurácia do EAT10 para identificar a disfagia. Foram estudados 120 pacientes internados com histórico de dificuldade de deglutição e 14 indivíduos saudáveis. A prevalência de disfagia foi de $87 \%$, sendo $75,6 \%$ com alteração na eficácia da deglutição e $80,9 \%$ com prejuízo da segurança da deglutição, dados esses, diferentes dos resultados desse estudo.

Quanto a avaliação do consumo alimentar, houve diferença estatística no consumo de frutas frescas e batata/salgados fritos, sendo que as mulheres apresentaram maior consumo de frutas frescas e os homens de batatas/salgados fritos. Figueiredo et al. (2008), em seu estudo com indivíduos adultos, verificou que as mulheres apresentaram maior frequência do consumo de frutas, legumes e verduras em comparação aos homens e a maior frequência do consumo desses alimentos ocorreu entre os indivíduos mais velhos. Em estudo epidemiológico realizado por Jaime e Monteiro (2005), também verificaram a influência positiva da idade sobre o consumo de frutas, legumes e verduras. Ainda, em relação ao consumo de batata/salgados fritos, segundo Bezerra et al. (2013), o consumo de alimentos fora do domicílio no Brasil dentre os homens apresentou médias totais de consumo superiores a das mulheres 
para a maioria dos grupos, com exceção de verduras, frutas e sopas. Gadenz e Benvegnu (2013) relataram em sua pesquisa com idosos hipertensos, conduzida em um município do interior do Rio Grande do Sul, que os idosos possuíam alimentação rica em gordura saturada e colesterol. Além disso, Santos e Ribeiro (2011) justificam que as práticas alimentares indicam muito da história cultural e social do indivíduo e correspondem aos procedimentos que vão desde a escolha, preparação até consumo do alimento. O comportamento alimentar de um indivíduo não envolve apenas a busca pela satisfação das necessidades fisiológicas, mas também as necessidades psicológicas, sociais e culturais que foram aprendidas na infância e podem se perpetuar ao longo da vida.

Este estudo possui como limitação a utilização do questionário de frequência alimentar que é qualitativo, não resultando nas quantidades de alimentos consumidos, sendo investigado apenas dez itens alimentares. Outra dificuldade encontrada é que foram encontrados poucos estudos utilizando questionários de avaliação da deglutição (EAT-10) e a autopercepção da saúde oral (GOHAI) relacionados com o consumo alimentar.

\section{Conclusão}

Não houve associação significativa entre os resultados da saúde oral com o consumo alimentar. Esses resultados são positivos, pois dentre os idosos analisados a maioria possui boas condições qualitativas para mastigação e deglutição. Por outro lado, em relação ao estado nutricional, encontrou-se maior obesidade abdominal em mulheres, dado este, que pode apontar risco para doenças cardiovasculares. Nesse sentido, outros estudos devem ser realizados para acompanhar se estes resultados se mantêm e o impacto sobre a saúde dos idosos.

Torna-se necessário reforçar orientações sobre alimentação saudável, para que os idosos possam adequar sua alimentação e prevenir problemas advindos de uma má nutrição, respeitando seus padrões alimentares e culturais, pois estes podem refletir na qualidade de vida dos idosos. Sugere-se a realização continua de outros estudos avaliando a saúde oral, a partir de instrumentos ou análises que comprovem as alterações e/ou problemas que possam afetar o consumo alimentar como forma de prevenção.

Os idosos avaliados nesse estudo residem na cidade de Veranópolis, que desde 1994 iniciou os estudos sobre o processo de envelhecimento e atualmente foi certificada pela Organização Mundial da Saúde como cidade amiga do idoso (WHO, 2016). 
Para obtenção da titularidade, os gestores do município, em parceria com o Centro Internacional de Longevidade Brasil (ILC) do Rio de Janeiro e o Centro de Pesquisa de Veranópolis, fizeram um levantamento com as principais necessidades dos idosos da cidade. Foram ouvidos 836 moradores com mais de 60 anos, sendo questionados sobre aspectos positivos e negativos abordando questões como: ambiente físico, moradia, transporte, participação, respeito e inclusão social, comunicação e informação, oportunidades de aprendizagem e apoio e cuidado. Com base nas solicitações, estão sendo propostas diversas ações, como oficina de informática, capacitação e atualização da equipe multiprofissional de saúde no atendimento ao idoso, com foco na prescrição, dispensação e orientação farmacêutica entre outros. Outro aspecto que se torna importante no nosso país, a exemplo do que acontece em Veranópolis, é a ampliação de estudos, pesquisa e políticas públicas para a inclusão dos idosos em seus municípios valorizando os saberes, as experiências, as trocas possíveis com os indivíduos em processo de envelhecimento. Além disso, é necessário incluir os idosos em grupos, universidades e programas de extensão, entre outros, para que eles possam participar ativamente, encontrar-se, incluir-se em atividades que compreendem lazer, cultura, saúde em especial a nutrição e alimentação. Nesse sentido, encontros onde se pode orientar que através da alimentação saudável é possível cuidar da saúde. Também, é necessário resgatar/valorizar preparações, receitas de família utilizando alimentos típicos e adequados aos idosos para manutenção da sua saúde e melhor qualidade de vida, sendo extensivo às suas famílias.

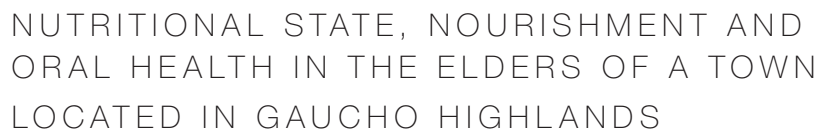

The goal of this study was to evaluate the nutritional sate, the nourishment and the oral health of elderly people in a town located in Gaucho Highlands, Rio Grande do Sul, Brazil. It was a transversal, descriptive, qualitative analytical, with primary data. The variations analysed were: gender, age ( $\geq 60$ years old), weight $(\mathrm{kg})$, height $(\mathrm{m})$, body mass index $\left(\mathrm{BM}=\mathrm{kg} / \mathrm{m}^{2}\right)$, waist circumference $(\mathrm{WC}=\mathrm{cm})$, arm circumference $(\mathrm{AC}=\mathrm{Cm})$, calf circumference $(\mathrm{CC}=\mathrm{Cm})$. To the evaluation, we used the "Mini Nutritional Assesment" (MNA), the food intake was evaluated by the Quizz of Nourishment Frequency (QNF), (SISVAN). For the 
oral health, we used the GOHAl (Geriatric Oral Health Assesment Index) and the EAT-10 (Eating Assesment Tool). From the 135 elders who were evaluated, $71.9 \%$ were women. The abdominal obesity was more important in women ( $\mathrm{p}=0.037)$ and the risk of innutrition by MNA about the prevalence of low weight, by BMI, raised with the age $(p=0.021$ e $p=0.039)$. For the other methods of nutritional evaluation, the differences weren't statistically significant. Still, by MNA there was a significant difference, where the women perceived themselves with better health ( $p=0.030)$. When investigating the oral health, there wasn't a significant difference (p>0.05). The consumption of fresh fruits ( $p=0.006$ ) was bigger among the women and the consumption of potatoes/fried snacks ( $p=0.001$ ) was bigger among the men. There wasn't a significant association between the results of the oral health and food consumption ( $p>0.05$ ). It is necessary to reinforce guidances about healthy nourishment, so the elders can adequate their nourishment and prevent problems that come from a bad nutrition, as well as other studies must be done with these variations.

keywords

Nutritional state. Nourishment. Oral health. Food consumption.

referências

ATCHISON, Kathryn; DOLAN, Teresa. Development of the geriatric oral health assessment index. Journal of Dental Education, Washington, DC, n. 54, p. 680-686, 1990.

ATLAS BRASIL. Atlas do desenvolvimento humano no Brasil, 2013. Disponível em: <http://www.atlasbrasil.org.br/2013/perfil/veranopolis_rs>. Acesso em: 18 out. 2015.

BASSLER Thais Carolina; LEI, Doris Lucia Martini. Diagnóstico e monitoramento da situação nutricional da população idosa em município da região metropolitana de Curitiba (PR). Revista de Nutrição, Campinas, v. 21, n. 3, p. 311-321, jun. 2008.

BELAFSKY, Peter et al. Validity and reliability of the Eating Assessment Tool (EAT-10). The Annals of Otology, Rhinology, and Laryngology, Los Angeles, v. 117, n. 12, p. 919-924, dez. 2008.

BEZERRA, llana Nogueira et al. Consumo de alimentos fora do domicílio no Brasil. Revista de Saúde Pública, São Paulo, v. 47, n. 1, p. 200-211, fev. 2013.

BRAZ, Marlene. A construção da subjetividade masculina e seu impacto sobre a saúde do homem: reflexão bioética sobre justiça distributiva. Ciência \& Saúde Coletiva, Rio de Janeiro, v. 10, n. 1, p. 97-104, jan. 2005.

CAMARGOS, Mirela Castro Santos; GONZAGA, Marcos Roberto. Viver mais e melhor? Estimativas de expectativa de vida saudável para a população brasileira. Cadernos de Saúde Pública, Rio de Janeiro, v. 31, n. 7, p. 1460-1472, jul. 2015. 
CAMPOS, Marta Alice Gomes et al. Estado nutricional e fatores associados em idosos. Revista da Associação Médica Brasileira, São Paulo, v. 52, n. 4, p. 214-221, jul. 2006.

CARVALHO, Catarina et al. Tradução e validação da versão portuguesa do Geriatric Oral Health Assessment Index (GOHAl). Revista Portuguesa de Saúde Pública, Lisboa, v. 31, n. 2, p. 166-172, jul./dez. 2013.

CASSOL, Karlla et al. Qualidade de vida em deglutição em idosos saudáveis. Jornal da Sociedade Brasileira de Fonoaudiologia, São Paulo, v. 24, n. 3, p. 223-232, 2012.

CRUZ, Ivana Beatrice Mânica da; MORIGUCHI, Emilio Hideyuki. Projeto Veranópolis: reflexos sobre envelhecimento bem sucedido. Porto Alegre: Oficina da Longevidade, 2002. p. 138

DYE, Bruce et al. Trends in oral health status: United States, 1988-1994 and 1999-2004. Vital Health Statistics, Hyattsville, v. 11, n. 248, p. 1-92, abr. 2007.

FELIX, Luciana Nabuco; SOUZA, Elizabeth Maria Talá de. Avaliação nutricional de idosos em uma instituição por diferentes instrumentos. Revista de Nutrição, Campinas, v. 22, n. 4, p. 571-580, jul./ago. 2009.

FIGUEIREDO, Iramaia Campos Ribeiro et al. Fatores associados ao consumo de frutas, legumes e verduras em adultos da cidade de São Paulo. Revista de Saúde Pública, São Paulo, v. 42, n. 5, p. 777-785, ago. 2008.

FUNDAÇÃO DE ECONOMIA E ESTATíTICA (FEE). Projeções populacionais antigas. Porto Alegre, nov. 2012. Disponível em: <http://www.fee.rs.gov.br/indicadores/populacao/ projecoes-populacionais/projecoes-populacionais-antigas/>. Acesso em: 4 jul. 2017.

GADENZ, Sabrina Dalbosco; BENVEGNU, Luís Antônio. Hábitos alimentares na prevenção de doenças cardiovasculares e fatores associados em idosos hipertensos. Ciência \& Saúde Coletiva, Rio de Janeiro, v. 18, n. 12, p. 3523-3533, dez. 2013.

GOMES, Romeu et al. Por que os homens buscam menos os serviços de saúde do que as mulheres? As explicações de homens com baixa escolaridade e homens com ensino superior. Cadernos de Saúde Pública, Rio de Janeiro, v. 23, n. 3, p. 565-74, mar. 2007.

INSTITUTO BRASILEIRO DE GEOGRAFIA E ESTATÍSTICA (IBGE). Agência de notícias. Em 2014, esperança de vida ao nascer era de 75,2 anos, Rio de Janeiro, 25 maio 2017. Disponível em: <http://saladeimprensa.ibge.gov.br/noticias? view=noticia\&id=1\& busca=1\&idnoticia=3045>. Acesso em: 4 jul. 2017.

Departamento de População e Indicadores Sociais. Perfil dos idosos responsáveis pelos domicilios. Rio de Janeiro: IBGE, 25 jul. 2002. Disponível em: <http:// www.ibge.gov.br/home/presidencia/noticias/25072002pidoso.shtm>. Acesso em: 4 jul. 2017.

JAIME, Patrícia Constante; MONTEIRO, Carlos Augusto. Consumo de frutas e hortaliças na população adulta brasileira, 2003. Cadernos de Saúde Pública, Rio de Janeiro, v. 21, n. 1, p. 19-24, 2005

LEWANDOWSKI, Andressa; BÓS Ângelo José Gonçalves. Estado de saúde bucal e necessidade de prótese dentária em idosos longevos. Revista da Associação Paulista de Cirurgiões Dentistas, São Paulo, v. 68, n. 2, p. 155-158, abr./jun. 2014.

LIPSCHITZ, David A. Screening for nutritional status in the elderly. Primare Care, Philadelphia, v. 21, n. 1, p. 55-67, mar. 1994.

MEDEIROS, Sofia Lince de; PONTES, Marília Pinheiro de Brito; MAGALHÃES, Hipólito Virgílio. Autopercepção da capacidade mastigatória em indivíduos idosos. Revista Brasileira de Geriatria e Gerontologia, Rio de Janeiro, v. 17, n. 4, p. 807-817, dez. 2014.

MIRANDA, Daniela Elias Goulart de Andrade et al. Manual de avaliação nutricional do adulto e do idoso. Rio de Janeiro: Rubio, 2012. 
MORAIS, Fernanda Tatiane Dias et al. Diagnóstico nutricional em idosos hospitalizados. Nutrir Gerais, Ipatinga, v. 4, n. 7, p. 637-651, ago./dez. 2010.

MORYA, Shingo et al. Relationships between self-assessed masticatory ability and higher level functional capacity among community-dwelling young-old persons. International Journal of Gerontology, Oxford, v. 6, n. 1, p. 33-37, mar. 2012.

NAJAS, Myrian Spinola; NEBULONI, Clarice C.. Avaliação Nutricional. In: RAMOS, L. R., TONIOLO NETO, J. Geriatria e Gerontologia. Barueri: Manole, 2005.

NASCIMENTO, Clarissa de Matos et al. Estado nutricional e fatores associados em idosos do Município de Viçosa, Minas Gerais, Brasil. Cadernos de Saúde Pública, Rio de Janeiro, v. 12, n. 7, p. 2409-2418, dez. 2011.

NUNES, Claudete Ivani Panizzi; ABEGG, Claíde. Factors associated with oral health perceptions in older Brazilians. Gerodontology, Mount Desert Island, v. 25, n. 1, p. 42-48, mar. 2008.

PALMER, Carole et al. It's More than Just Candy: important relationships between nutrition and oral health. Nutrition Today, Baltimore, v. 45, n. 4, p. 154-166, jul./ago. 2010.

PERELMAN, Julian; FERNANDES, Ana; MATEUS, Céu. Disparidades de gênero na saúde e nos cuidados de saúde: resultados para Portugal com base no Inquérito Nacional de Saúde. Cadernos de Saúde Pública, Rio de Janeiro, v. 28, n. 12, p. 2339-2348, dez. 2012

ROFES, Laia et al. Sensitivity and specificity of the Eating Assessment Tool and the Volume-Viscosity Swallow Test for clinical evaluation of oropharyngeal dysphagia. Neurogastroenterology and Motility, Osney Mead, v. 26, n. 9, p. 1256-1265, set. 2014.

ROSSI, Luciana et al. Avaliação Nutricional: novas perspectivas. São Paulo: Roca, 2008.

SANTOS, Glenda Dias dos; RIBEIRO, Sandra Maria Lima. Aspectos afetivos relacionados ao comportamento alimentar dos idosos frequentadores de um centro de convivência. Revista Brasileira de Geriatria e Gerontologia, Rio de Janeiro, v. 14, n. 2 , p. 319-328, jun. 2011.

SILVA, Débora Dias da et al. Autopercepção e condições de saúde bucal em uma população de idosos. Cadernos de Saúde Pública, Rio de Janeiro, v. 21, n.4, p. 1251 1259, jul./ago.2005.

SILVA, Silvio Rocha Côrrea da; CASTELLANOS, Roberto Fernandes. Autopercepção das condições de saúde bucal por idosos. Revista de Saúde Pública, São Paulo, v. 35, n. 4, p. 349-355, 2001.

SPEROTTO, Francieli Marina; SPINELLI, Roseana Baggio. Avaliação nutricional em idosos independentes de uma instituição de longa permanência no município de Erechim - RS. Revista Perspectiva, Erechim, v. 34, n. 125, p. 105-116, mar. 2010.

SPOSITO, Andrei Carvalho. IV Diretriz Brasileira sobre Dislipidemias e Prevenção da Aterosclerose. Arquivos Brasileiros de Cardiologia, São Paulo, v. 88, p. 2-19, abr. 2007. Suplemento.

TRAMONTINO, Vanessa Silva et al. Nutrição para idosos. Revista de Odontologia da Universidade Cidade de São Paulo, São Paulo, v. 21, n. 3, p. 258-267, set./dez. 2009.

VAN DER BILT, Andries. Assessment of mastication with implications for oral rehabilitation: a review. Journal of Oral Rehabilitation, Oxford, v. 38, n. 10, p. 754-780, out. 2011

VENTURINI, Carina Duarte et al. Prevalência de obesidade associada à ingestão calórica, glicêmica e perfil lipídico em uma amostra populacional de idosos do Su do Brasil. Revista Brasileira de Geriatria e Gerontologia, Rio de Janeiro, v. 16, n. 3, p. 591-601, jul./set. 2013. 
WACHHOLZ, Patrick Alexander; RODRIGUES, Sueleen Cristiane; YAMANE, Roseli. Estado nutricional e a qualidade de vida em homens idosos vivendo em instituição de longa permanência em Curitiba, PR. Revista Brasileira de Geriatria e Gerontologia, Rio de Janeiro, v. 14, n. 4, p. 625-635, out./dez. 2011.

WORLD HEALTH ORGANIZATION (WHO). Age-Friendly World: Veranópolis, 2016. Disponível em: <https://extranet.who.int/agefriendlyworld/network/veranopolis>. Acesso em: 2 jun. 2017.

Guia para o Dia Mundial da Saúde, Angola, 7 abr. 2012. Disponível em: <http:// www.afro.who.int/pt/angola/press-materials/item/4452-guia-para-o-dia-mundial-da-sa\%C3\%BAde-7-de-abril-de-2012.html>. Acesso em: 7 abr. 2014

Data de submissão: 25/11/2015

Data de aprovação: 16/06/2017 